Mathematical Modelling and Analysis

Volume 22 Number 4, July 2017, 464-483

https://doi.org/10.3846/13926292.2017.1323035

(c) Vilnius Gediminas Technical University, 2017
Publisher: Taylor\&Francis and VGTU

http://www.tandfonline.com/TMMA

ISSN: $1392-6292$

eISSN: $1648-3510$

\title{
Comparison of Closed-Form Solutions for the Lucas-Uzawa Model via the Partial Hamiltonian Approach and the Classical Approach
}

\author{
Rehana $\mathrm{Naz}^{a}$ and Azam Chaudhry ${ }^{b}$ \\ ${ }^{a}$ Lahore School of Economics \\ Centre for Mathematics and Statistical Sciences, 53200 Lahore, Pakistan \\ ${ }^{b}$ Lahore School of Economics \\ Department of Economics, 53200 Lahore, Pakistan \\ E-mail(corresp.): rehananaz_qau@yahoo.com \\ E-mail: drrehana@lahoreschool.edu.pk \\ E-mail: azam@lahoreschool.edu.pk
}

Received December 21, 2016; revised April 18, 2017; published online July 15, 2017

\begin{abstract}
In this paper we derive the closed-form solutions for the Lucas-Uzawa growth model with the aid of the partial Hamiltonian approach and then compare our results with those derived in literature. The partial Hamiltonian approach provides two first integrals [9] in the case where there are no parameter restrictions and these two first integrals are utilized to construct three sets of closed form solutions for all the variables in the model. We begin by using the two first integrals to find two closed form solutions, one of which is new to the literature. We then use only one of the first integrals to derive a third solution that is the same as that found in the previous literature. We continue by analyzing the newly derived solution in detail also show that all three solutions converge to the same long run balanced growth path. The special case when the share of capital is equal to the inverse of the intertemporal elasticity of substitution is also investigated in detail.
\end{abstract}

Keywords: economic growth, partial Hamiltonian approach, Lucas-Uzawa model, currentvalue Hamiltonian.

AMS Subject Classification: 35L65; 76M60; 83C15.

\section{Introduction}

One of the foundations of modern economic growth theory is the two-sector endogenous growth model developed by Lucas and Uzawa. The model addresses the relationship between human capital accumulation and economic growth and the idea behind the Lucas-Uzawa model ( [5] and [11]) is to determine optimal time paths for consumption and the amount of labor devoted to the production of capital in an economy which has constrained levels of physical 
and human capital. One of the interesting features of the Lucas-Uzawa model is the existence of multiple equilibria which implies different steady state values for key economic variables in the model.

The partial Hamiltonian approach [10] uses tools from Lie group theory and is used to construct closed-form solutions of dynamical systems such as those arising in economic growth theory. This approach is unique and a significant departure from the rest of the literature because unlike the previously used methods, the partial Hamiltonian is applicable to an arbitrary system of ordinary differential equations which means that it can be applied to more complex models [8] in economic growth and other fields. In the context of our paper, the partial Hamiltonian methodology yields a series of first integrals for a system of ordinary differential equations and we use these first integrals to find closed-form solutions for the Lucas-Uzawa model of economic growth. In this paper we establish the closed-form solutions for the Lucas-Uzawa model with the aid of the partial Hamiltonian approach and we compare our results with those derived by the classical approach $[1,3]$. The partial Hamiltonian approach provides two first integrals [9] for case where there are no parameter restrictions. We utilize these two first integrals to construct closed form solutions for all variables of model for two different scenarios: (i) $z=z^{*}$ and (ii) $z \neq z^{*}$ where $z(t)=\frac{h(t) u(t)}{k(t)}$ where $k(t)$ is physical capital, $h(t)$ is human capital and $u(t)$ is the fraction of labor devoted to the production of physical capital.

We begin by using both first integrals to construct closed-form solutions for both scenarios. One solution is exactly the same as derived by Chilarescu [3] and the second is completely new to the literature. We then use only one first integral to determine a different solution, again under fairly general parameter values, and show that this is the same solution that has been derived in the previous literature (see $[1,3]$ ).

We find that in the case where $z=z^{*}$, both the partial Hamiltonian approach and the classical approach provide one solution. For the $z \neq z^{*}$ case, the classical approach yields one solution while the partial Hamiltonian approach yields the same solution as well as providing one additional solution which is completely new to the economic growth literature. What is especially interesting about our new solution is that while the equilibrium levels of consumption and capital stock in the new solution are equal to those found in the old solution, the amount of labor allocated to the production of physical capital and the level of human capital are different in the new solution. In the context of economic growth theory, this means that an economy can be in either a high or low human capital equilibrium. The existence of three closed form solutions is new to the literature and we also show that these closed form solutions all converge to the same long run balanced growth path.

It is important to mention here that under a specific parameter restriction $\sigma=\frac{\beta(\rho+\pi)}{2 \pi \beta-\delta+\delta \beta-\pi}$ (where $\sigma$ is the inverse of the elasticity of intertemporal substitution) a third first integral was obtained and the closed form solution for this case was new in the literature (see Naz et al [9]). The partial Hamiltonian approach provides three solutions for the case in which there are no parameter restrictions and fourth solution for the specific parameter restric- 
tion $\sigma=\frac{\beta(\rho+\pi)}{2 \pi \beta-\delta+\delta \beta-\pi}$ was established in Naz et al [9]. The classical approach provides only two closed-form solutions for this model (see Chilarescu [3]) which means that the partial Hamiltonian approach not only provides all the solutions constructed in the previous literature (see Chilarescu [3]) but also provides additional closed-form solutions which are completely new to the literature on economic growth. Moreover, the special case of when the share of capital is equal to the inverse of the intertemporal elasticity of substitution is also discussed in detail. We then analyze our results in the context of the previous literature by comparing our results with those found by Marsiglio and La Torre $[6,7]$ who derived closed form solutions for the model under various types of population growth.

The layout of the paper is as follows. In Section 2, we provide an overview of partial Hamiltonian approach. Moreover, we introduce the Lucas-Uzawa model and provided an overview of first integrals derived by partial Hamiltonian approach previously in [9]. In Section 3, the closed-form solutions of the dynamical system of ODEs are constructed by utilizing both first integrals in the case of no parameter restrictions. In Section 4, we use only one first integral to derive the closed form solutions for all the variables in the model. A comparison of our results with those derived by the classical approach is presented in Section 5. In Section 6, the closed-form solutions of the dynamical system of ODEs are provided for $\sigma=\beta$. Finally, our conclusions are summarized in Section 7 .

\section{Overview of papers $[9,10]$}

In this section, we provide an overview of partial Hamiltonian approach [10]. Moreover, the overview of first integrals for Lucas-Uzawa model derived by partial Hamiltonian approach [9] is presented.

\subsection{Overview of partial Hamiltonian approach}

Let time $t$ be the independent variable and $(q, p)=\left(q^{1}, \ldots, q^{n}, p_{1}, \ldots, p_{n}\right)$ the phase space coordinates. We provide an overview of partial Hamiltonian approach $[9,10]$. The following definitions and results are adapted from $[2,4,9,10]$. The total derivative operator with respect to the time $t$ is given by

$$
D=\frac{\partial}{\partial t}+\dot{q}^{i} \frac{\partial}{\partial q^{i}}+\dot{p}_{i} \frac{\partial}{\partial p_{i}}+\ldots
$$

The Euler operator $\delta / \delta q^{i}$ is defined as

$$
\frac{\delta}{\delta q^{i}}=\frac{\partial}{\partial q^{i}}-D \frac{\partial}{\partial \dot{q}^{i}}, i=1,2, \ldots, n
$$

and the variational operator $\delta / \delta p_{i}$ is given by

$$
\frac{\delta}{\delta p_{i}}=\frac{\partial}{\partial p_{i}}-D \frac{\partial}{\partial \dot{p}_{i}}, i=1,2, \ldots, n \text {. }
$$


The summation convention applies for repeated indices.

The variables $t, q, p$ are independent and satisfy

$$
\dot{p}_{i}=D\left(p_{i}\right), \dot{q}^{i}=D\left(q^{i}\right), i=1,2, \ldots, n .
$$

In economic analysis, the optimal control problem for constant discount factor case, is stated as (see, e.g. [2])

$$
\begin{aligned}
\text { Maximize } \mathcal{F} & =\int_{0}^{T} F\left(t, q^{i}, c_{i}\right) \mathrm{e}^{-\rho t} d t, \\
\text { subject to } \dot{q}^{i} & =f^{i}\left(t, q^{i}, c_{i}\right), \quad i=1, \ldots, n,
\end{aligned}
$$

subject to appropriate initiate and transversality conditions. One can define minimization problems as well.

The current value Hamiltonian $H\left(t, q^{i}, p_{i}, c_{i}\right)$ (see, e.g. [2]) is defined as

$$
H=F\left(t, q^{i}, c_{i}\right)+p_{i} f^{i}\left(t, q^{i}, c_{i}\right) .
$$

The Pontryagin's maximum principle provides necessary conditions for optimal control for the current value Hamiltonian $H$ defined in (2.1) (see, e.g. [2])

$$
\begin{aligned}
& \frac{\partial H}{\partial c_{i}}=0, \quad \frac{\partial^{2} H}{\partial c_{i}{ }^{2}}<0, \\
& \dot{q}^{i}=\frac{\partial H}{\partial p_{i}}, \quad \dot{p}^{i}=-\frac{\partial H}{\partial q^{i}}+\Gamma_{i}, \quad i=1, \ldots, n,
\end{aligned}
$$

where $\Gamma_{i}=\Gamma_{i}\left(t, p_{i}, q^{i}\right)$ but for a constant discount factor $\mathrm{e}^{-\rho t}$, we have $\Gamma_{i}=$ $\rho p_{i}$. One can compute control variables $c_{i}=h_{i}\left(p_{i}, q^{i}\right)$ from first equation of condition of maximum principle for current value Hamiltonian.

The following result is adapted from [10] and is essential for construction of first integrals via partial Hamiltonian approach:

Theorem 1. (see [10] ) An operator $X$ of the form

$$
X=\xi\left(t, q^{i}, p_{i}\right) \frac{\partial}{\partial t}+\eta^{i}\left(t, q^{i}, p_{i}\right) \frac{\partial}{\partial q^{i}}+\zeta_{i}\left(t, q^{i}, p_{i}\right) \frac{\partial}{\partial p_{i}}
$$

is said to be a partial Hamiltonian operator corresponding to a current value Hamiltonian $H\left(t, q^{i}, p_{i}, c_{i}\right)$ which satisfies (2.2), if there exists a function $B\left(t, q^{i}, p_{i}\right)$ such that

$$
\zeta_{i} \frac{\partial H}{\partial p_{i}}+p_{i} D\left(\eta^{i}\right)-X(H)-H D(\xi)=D(B)+\left(\eta^{i}-\xi \frac{\partial H}{\partial p_{i}}\right)\left(-\Gamma_{i}\right)
$$

holds. The first integral corresponding to the system (2.2) associated with a partial Hamiltonian operator $X$ of the current value Hamiltonian $H\left(t, q^{i}, p_{i}, c_{i}\right)$ is determined from

$$
I=p_{i} \eta^{i}-\xi H-B,
$$

where $B\left(t, p_{i}, q^{i}\right)$ is a gauge-like function. 


\subsection{Overview of first integrals for the Lucas-Uzawa model via partial Hamiltonian approach}

In this section, we provide an overview of the partial Hamiltonian approach to solving the Lucas-Uzawa model which is taken from Naz et al [9].

The representative agent's utility function is defined as

$$
\operatorname{Max}_{c, u} \int_{0}^{\infty} \frac{c^{1-\sigma}-1}{1-\sigma} e^{-\rho t}, \sigma \neq 1,
$$

subject to the constraints of physical capital and human capital (see details of parameters:

$$
\begin{aligned}
& \dot{k}(t)=\gamma k^{\beta} u^{1-\beta} h^{1-\beta}-\pi k-c, k_{0}=k(0), \\
& \dot{h}(t)=\delta(1-u) h, h_{0}=h(0),
\end{aligned}
$$

where $1 / \sigma$ is the constant elasticity of intertemporal substitution, $\rho>0$ is the discount factor, $\beta$ is the elasticity of output with respect to physical capital, $\gamma>0$ is the technological levels in the goods sector, $\delta>0$ is the technological levels in the education sector, $k$ is the level of physical capital, $h$ is the level of human capital, $c$ is per capita consumption and $u$ is the fraction of labor allocated to the production of physical capital.

The current value Hamiltonian function is defined as

$$
H(t, c, k, \lambda)=\frac{c^{1-\sigma}-1}{1-\sigma}+\lambda\left[\gamma k^{\beta} u^{1-\beta} h^{1-\beta}-\pi k-c\right]+\mu \delta(1-u) h
$$

where $\lambda(t)$ and $\mu(t)$ are costate variables. The transversality conditions are

$$
\lim _{t \rightarrow \infty} e^{-\rho t} \lambda(t) k(t)=0, \lim _{t \rightarrow \infty} e^{-\rho t} \mu(t) h(t)=0 .
$$

The Pontryagin's maximum principle provides following set of first order conditions:

$$
\begin{aligned}
& \lambda=c^{-\sigma}, \\
& u^{\beta}=\frac{\gamma(1-\beta) k^{\beta} h^{-\beta}}{\delta} \frac{\lambda}{\mu}, \\
& \dot{k}(t)=\gamma k^{\beta} u^{1-\beta} h^{1-\beta}-\pi k-c, \\
& \dot{h}(t)=\delta(1-u) h, \\
& \dot{\lambda}=-\lambda \gamma \beta u^{1-\beta} k^{\beta-1} h^{1-\beta}+\lambda(\rho+\pi), \\
& \dot{\mu}=\mu(\rho-\delta) .
\end{aligned}
$$

The growth rates of consumption $c$ and physical capital $u$ are given by

$$
\begin{aligned}
& \frac{\dot{c}}{c}=\frac{\beta \gamma}{\sigma} u^{1-\beta} k^{\beta-1} h^{1-\beta}-\frac{\rho+\pi}{\sigma}, \\
& \frac{\dot{u}}{u}=\frac{(\delta+\pi)(1-\beta)}{\beta}-\frac{c}{k}+\delta u .
\end{aligned}
$$


The partial Hamiltonian determining equation (2.3), for the current value (partial) Hamiltonian (2.6), yields

$$
\begin{aligned}
& \lambda\left(\eta_{t}^{1}+\dot{k} \eta_{k}^{1}+\dot{h} \eta_{h}^{1}\right)+\mu\left(\eta_{t}^{2}+\dot{k} \eta_{k}^{2}+\dot{h} \eta_{h}^{2}\right)-\eta^{1}\left[\beta \lambda \gamma(h u / k)^{1-\beta}-\pi \lambda\right] \\
& \quad-\eta^{2} \delta \mu-H\left(\xi_{t}+\dot{k} \xi_{k}+\dot{h} \xi_{h}\right)=B_{t}+\dot{k} B_{k}+\dot{h} B_{h} \\
& +\left[\eta^{1}-\xi\left(\gamma k^{\beta}(u h)^{1-\beta}-\pi k-c\right)\right](-\lambda \rho)+\left[\eta^{2}-\xi \delta(1-u) h\right](-\mu \rho),
\end{aligned}
$$

in which we assume that $\xi=\xi(t, k, h), \eta^{1}(t, k, h), \eta^{2}(t, k, h), B=B(t, k, h)$. Note that for Lucas-Uzawa model $q^{1}=k, q^{2}=h, p_{1}=\lambda, p_{2}=\mu, \Gamma_{1}=$ $\rho \lambda, \Gamma_{2}=\rho \mu$. The variables $c$ (consumption) and $u$ (fraction of labor devoted to producing physical capital) are control variable which are connected with co-state variables $\lambda, \mu$ by equations (2.8) and (2.9). It is worthy to mention here that in partial Hamiltonian operator determining equation (2.16) either we can keep control variables $c$ and $u$ or co-state variables $\lambda, \mu$ as these are connected by equations (2.8) and (2.9). First, we expand (2.16) and then we eliminate $\lambda, \mu$ by utilizing (2.8) and (2.9). The resulting equation is then separated with respect to powers of the control variables $c$ and $u$, we have

$$
\begin{aligned}
c^{2-\sigma}: & \xi_{k}=0, \\
c^{-\sigma} u^{2-\beta}: & \xi_{h}=0, \\
u c^{-\sigma}: & \eta_{h}^{1}=0 \\
c^{1-\sigma}: & -\eta_{k}^{1}-\sigma \xi_{t} /(1-\sigma)+\rho \xi=0, \\
c^{1-\sigma} u^{-\beta}: & \eta_{k}^{2}=0, \\
c^{-\sigma} u^{1-\beta}: & \eta_{k}^{1}-(1-\beta) \eta_{h}^{2}-\eta^{1} \frac{\beta}{k}-\beta \xi_{t}-\rho \xi \beta=0, \\
c^{-\sigma}: & \eta_{t}^{1}-\pi k \eta_{k}^{1}+\eta^{1} \pi+\pi k \xi_{t}+\rho \eta^{1}+\rho \pi k \xi=0, \\
c^{-\sigma} u^{-\beta}: & \eta_{t}^{2}+\delta h \eta_{h}^{2}-\delta \eta^{2}-\delta h \xi_{t}+\rho \eta^{2}-\rho \xi \delta h=0, \\
c: & B_{k}=0, \\
u: & B_{h}=0, \\
1: & B_{t}=\xi_{t} /(1-\sigma) .
\end{aligned}
$$

The solution of equations (2.17)-(2.27) yield the expressions for $\xi, \eta^{1}, \eta^{2}$ and $B$. For the case, when there is no restriction on the parameters the partial Hamiltonian operators and gauge terms are given by

$$
\begin{aligned}
& \xi=0, \eta^{1}=0, \eta^{2}=e^{-(\rho-\delta) t}, B(t)=0, \\
& \xi=e^{-\rho t}, \eta^{1}=\frac{1}{1-\sigma} \rho e^{-\rho t} k, \eta^{2}=\frac{1}{1-\sigma} \rho e^{-\rho t} h, \quad B=\frac{1}{1-\sigma} e^{-\rho t} .
\end{aligned}
$$

Under the parameters restriction $\sigma=\frac{\beta(\rho+\pi)}{-\pi+2 \pi \beta-\delta+\delta \beta}$, the partial Hamiltonian operators and gauge term are given by

$$
\begin{aligned}
\xi & =e^{-\frac{(\sigma-1)(\rho+\pi)}{\sigma} t}, \eta^{1}=-\pi k e^{-\frac{(\sigma-1)(\rho+\pi)}{\sigma} t}, \\
\eta^{2} & =\frac{h}{\sigma(1-\beta)}[-\rho \beta-\pi \sigma+2 \beta \sigma \pi-\pi \beta] e^{-\frac{(\sigma-1)(\rho+\pi)}{\sigma} t},
\end{aligned}
$$




$$
B=e^{-\frac{(\sigma-1)(\rho+\pi)}{\sigma} t} /(1-\sigma) \text {. }
$$

For the partial Hamiltonian operators and gauge terms given in (2.28), formula (2.4) yields following two first integrals with no restrictions on parameters of economy:

$$
\begin{aligned}
I_{1}= & \frac{\gamma(1-\beta)}{\delta} c^{-\sigma} k^{\beta} u^{-\beta} h^{-\beta} e^{-(\rho-\delta) t}, \\
I_{2}= & \frac{c^{-\sigma} e^{-\rho t}}{1-\sigma}\left[(\rho+\pi-\pi \sigma) k-\sigma c-\beta \gamma(1-\sigma)\left(\frac{u h}{k}\right)^{1-\beta} k\right. \\
& \left.+\frac{(1-\beta) \gamma}{\delta}(\rho-\delta+\delta \sigma)\left(\frac{k}{u h}\right)^{\beta} h\right] .
\end{aligned}
$$

Under parameter restriction $\sigma=\frac{\beta(\rho+\pi)}{2 \pi \beta-\delta+\delta \beta-\pi}$, the formula (2.4) with the aid of the partial Hamiltonian operators and gauge terms given in (2.29) provides following first integral:

$$
I_{3}=e^{-\frac{(\sigma-1)(\rho+\pi)}{\sigma} t} c^{-\sigma}\left[-\frac{\beta(\rho+\pi)}{(\pi+\delta-\rho) \beta-\pi-\delta} c-\beta \gamma\left(\frac{u h}{k}\right)^{1-\beta} k\right],
$$

provided $2 \pi \beta-\delta+\delta \beta-\pi>0$ to ensure that $\sigma>0$.

The complete analysis in terms of closed-from solution under parameter restriction $\sigma=\frac{\beta(\rho+\pi)}{2 \pi \beta-\delta+\delta \beta-\pi}$ was provided by Naz et al [9]. The convergence of closed-form solution to balanced growth path was established as well.

\section{Closed-form solution for the Lucas-Uzawa model under fairly general values of parameters via $I_{1}$ and $I_{2}$}

The closed-form solution via $I_{1}$ and $I_{2}$ for the case $z \neq z^{*}$ case was provided in Naz et al [9] but was not analyzed in detail. Here, we provide closed form solutions for the original economic variables $c(t), u(t), k(t), h(t), \lambda(t)$ and $\mu(t)$ for the case $z \neq z^{*}$ explicitly in terms of variable $z(t)$. Moreover, we provide closed form solutions for the original variables $c(t), u(t), k(t), h(t), \lambda(t)$ and $\mu(t)$ for the case $z=z^{*}$ as well. We also compare our solution with the previous literature.

By setting $I_{1}=c_{1}$, we obtain

$$
\frac{\gamma(1-\beta)}{\delta} c^{-\sigma} k^{\beta} u^{-\beta} h^{-\beta} e^{-(\rho-\delta) t}=c_{1}
$$

where $c_{1}$ is an arbitrary constant. Introducing $z=\frac{h u}{k}$, Equation (3.1) can be re-written as

$$
z=\left(\frac{(1-\beta) \gamma}{c_{1} \delta}\right)^{\frac{1}{\beta}} \lambda^{\frac{1}{\beta}} e^{-\frac{(\rho-\delta)}{\beta} t}
$$

where $\lambda=c^{-\sigma}$. Equation (2.12) with the aid of Equation (3.2) yields following Bernoulli's differential equation for $\lambda$

$$
\dot{\lambda}-\lambda(\rho+\pi)=-\beta \gamma\left[\frac{(1-\beta) \gamma}{c_{1} \delta}\right]^{\frac{1-\beta}{\beta}} \lambda^{\frac{1}{\beta}} e^{-\frac{(\rho-\delta)(1-\beta)}{\beta} t},
$$


and thus we have

$$
\lambda=c^{-\sigma}=\left[\frac{\beta \gamma^{\frac{1}{\beta}}}{\delta+\pi}\left(\frac{1-\beta}{c_{1} \delta}\right)^{\frac{1-\beta}{\beta}} e^{-\frac{(\rho-\delta)(1-\beta)}{\beta} t}+c_{2} e^{-\frac{(1-\beta)(\rho+\pi)}{\beta} t}\right]^{\frac{\beta}{\beta-1}},
$$

where $c_{2}$ is an arbitrary constant. We found explicit solution for $z$ after substituting value of $\lambda$ from Equation (3.3) into Equation (3.2)

$$
z=\left[\frac{\beta \gamma}{\delta+\pi}+c_{2}\left(\frac{(1-\beta) \gamma}{c_{1} \delta}\right)^{\frac{\beta-1}{\beta}} e^{-\frac{(1-\beta)(\delta+\pi)}{\beta} t}\right]^{\frac{1}{\beta-1}}
$$

and $z^{*}=(\beta \gamma /(\delta+\pi))^{1 /(\beta-1)}$ is the steady state solution. It is worthy to mention here that the system of differential equations (2.10)-(2.13) provides two sets of solutions depending on $c_{2}=0$ and $c_{2} \neq 0$.

Thus we discuss two scenarios. Scenario I: $c_{2}=0$ and thus $z=z^{*}$. Scenario II: $c_{2} \neq 0$ and thus $z \neq z^{*}$.

\subsection{Scenario I: $c_{2}=0$ and thus $z=z^{*}$}

For $c_{2}=0$, we have

$$
z(t)=\left(\frac{\delta+\pi}{\beta \gamma}\right)^{\frac{1}{1-\beta}}=z^{*}
$$

Equation (3.2) yields

$$
\lambda(t)=\frac{c_{1} \delta}{(1-\beta) \gamma} e^{(\rho-\delta) t} z^{* \beta}
$$

and thus

$$
c(t)=\left(\frac{(1-\beta) \gamma z^{* \beta}}{c_{1} \delta}\right)^{\frac{1}{\sigma}} e^{-\frac{(\rho-\delta)}{\sigma} t} .
$$

Using initial condition $c(0)=c_{0}$ and $k(0)=k_{0}$, Equation (3.7) gives

$$
c_{0}=\left(\frac{(1-\beta) \gamma}{c_{1} \delta z^{* \beta}}\right)^{\frac{1}{\sigma}} \text {. }
$$

Equation (2.10) provides following solution for physical capital $k(t)$ :

$$
k(t)=\frac{\sigma \beta c_{0}}{\sigma \pi+\delta(\sigma-\beta)-(\pi \sigma-\rho) \beta} e^{-\frac{(\rho-\delta)}{\sigma} t}+a_{1} e^{-\frac{(\pi \beta-\pi-\delta)}{\beta} t} .
$$

The transversality condition (2.7) for $k$ is satisfied provided $\delta<\rho+\delta \sigma$ and $a_{1}=0$. Using initial condition $k(0)=k_{0}$, we have

$$
\frac{c_{0}}{k_{0}}=\frac{\delta+\pi(1-\beta)}{\beta}-\frac{\delta-\rho}{\sigma}>0,
$$

as $\frac{\delta+\pi-\pi \beta}{\beta}-\frac{\delta-\rho}{\sigma}>0$ (see proof in Proposition 1 [3]). Equations (2.9) and (3.1) provide following expression of costate variable $\mu$

$$
\mu=c_{1} e^{(\rho-\delta) t} .
$$


Next, we set $I_{2}=c_{3}$ and after some simplifications, we arrive at following expression for human capital $h$

$$
\begin{aligned}
h(t)= & \frac{\delta}{\gamma(1-\beta)(\rho-\delta+\delta \sigma)} z^{* \beta}\left[c_{3}(1-\sigma) c_{0}^{\sigma} e^{-\delta t}\right. \\
& \left.+\left(\beta \gamma(1-\sigma) z^{* 1-\beta} k_{0}-(\rho+\pi-\pi \sigma) k_{0}+\sigma c_{0}\right) e^{-\frac{(\rho-\delta)}{\sigma} t}\right] .
\end{aligned}
$$

The transversality condition (2.7) for $h$ is satisfied provided $c_{3}=0$ and $\delta<\rho+\delta \sigma$. The initial condition $h(0)=h_{0}$ yields

$$
h(t)=h_{0} e^{-\frac{(\rho-\delta)}{\sigma} t}, \quad h_{0}=\frac{z^{*} k_{0} \delta \sigma}{\rho-\delta+\delta \sigma} .
$$

Finally, we obtain an expression for the fraction of labor devoted to the production of physical capital $u=\frac{z k}{h}$

$$
u=(\rho-\delta+\delta \sigma) /(\delta \sigma)
$$

and this completes the solution. We can summarize these solutions for all the economic variables in the following simple forms:

$$
\begin{aligned}
& c(t)=c_{0} e^{-\frac{(\rho-\delta)}{\sigma} t}, \quad k(t)=k_{0} e^{-\frac{(\rho-\delta)}{\sigma} t}, \\
& u(t)=\frac{\rho-\delta+\delta \sigma}{\delta \sigma}=u^{*}, \quad h(t)=h_{0} e^{-\frac{(\rho-\delta)}{\sigma} t}, \\
& \lambda(t)=c_{0}^{-\sigma} e^{(\rho-\delta) t}, \quad \mu=c_{1} e^{(\rho-\delta) t}, \quad z^{*}=\left(\frac{\delta+\pi}{\beta \gamma}\right)^{\frac{1}{1-\beta}},
\end{aligned}
$$

provided $\delta<\rho+\delta \sigma, c_{0}=\left(\frac{(1-\beta) \gamma}{c_{1} \delta z^{* \beta}}\right)^{\frac{1}{\sigma}}, \frac{c_{0}}{k_{0}}=\frac{\delta+\pi(1-\beta)}{\beta}-\frac{\delta-\rho}{\sigma}>0, h_{0}=\frac{z^{*} k_{0}}{u^{*}}$.

\subsection{Scenario II: $c_{2} \neq 0$ and thus $z \neq z^{*}$}

The expression for $\lambda$ given in Equation (3.3) can be alternatively given as

$$
\lambda=\frac{c_{1} \delta}{(1-\beta) \gamma} e^{(\rho-\delta) t} z^{\beta} .
$$

Equation (3.4) for $z(t)$ with initial condition $z(0)=z_{0}$ takes following form:

$$
z(t)=\frac{z^{*} z_{0}}{\left[\left(z^{* 1-\beta}-z_{0}^{1-\beta}\right) e^{-\frac{(1-\beta)(\delta+\pi)}{\beta} t}+z_{0}^{1-\beta}\right]^{\frac{1}{1-\beta}}},
$$

where $c_{2}=\left(z_{0}^{1-\beta}-z^{* 1-\beta}\right) /\left(\frac{(1-\beta) \gamma}{c_{1} \delta}\right)^{\frac{\beta-1}{\beta}}$.

The variable $c(t)$ with $c(0)=c_{0}$ takes following form:

$$
c=c_{0} z_{0}^{\frac{\beta}{\sigma}} e^{-\frac{(\rho-\delta)}{\sigma} t} z^{-\frac{\beta}{\sigma}}, c_{0} z_{0}^{\frac{\beta}{\sigma}}=\left(\frac{c_{1} \delta}{(1-\beta) \gamma}\right)^{-\frac{1}{\sigma}}
$$


where $z$ is same as given in (3.13). The differential equation (2.10) for $k$ results in following integrable differential equation

$$
\dot{k}+\left(\pi-\gamma z^{1-\beta}\right) k=-c_{0} z_{0}^{\frac{\beta}{\sigma}} e^{-\frac{(\rho-\delta)}{\sigma} t} z^{-\frac{\beta}{\sigma}}
$$

and it provides an expression for physical capital

$$
k(t)=\left(a_{3}-\frac{c_{0} z_{0}^{\frac{\beta}{\sigma}}}{(\pi+\delta)^{\frac{1}{1-\beta}}} F(t)\right)(\pi+\delta)^{\frac{1}{1-\beta}} z(t)^{-1} e^{\frac{(\pi+\delta-\pi \beta)}{\beta} t},
$$

where

$$
F(t)=\int_{0}^{t} z(t)^{\frac{\sigma-\beta}{\sigma}} e^{-\left(\frac{\delta+\pi-\pi \beta}{\beta}-\frac{\delta-\rho}{\sigma}\right) t} d t
$$

$a_{3}$ is arbitrary constant of integration and $\frac{\delta+\pi-\pi \beta}{\beta}-\frac{\delta-\rho}{\sigma}>0$ (see proof in Proposition 1 [3]). The initial condition $k(0)=k_{0}$ yields $a_{3}=\frac{k_{0} z_{0}}{(\pi+\delta)^{\frac{1}{1-\beta}}}$ and thus expression for $k(t)$ simplifies to the following form:

$$
k(t)=\left(k_{0} /\left(c_{0} z_{0}^{\frac{\beta-\sigma}{\sigma}}\right)-F(t)\right) c_{0} z_{0}^{\frac{\beta}{\sigma}} z(t)^{-1} e^{\frac{(\delta+\pi-\pi \beta)}{\beta} t} .
$$

The transversality condition (2.7) for $k$ is satisfied provided $\delta<\rho+\delta \sigma$ and

$$
\lim _{t \rightarrow \infty} F(t)=k_{0} /\left(c_{0} z_{0}^{(\beta-\sigma) / \sigma}\right) .
$$

It is important to mention here that the integrand of $F(t)$ is positive and bounded therefore $\lim _{t \rightarrow \infty} F(t)$ is a finite number. Equations (2.9) and (3.1) provide following expression for the costate variable

$$
\mu=c_{1} e^{(\rho-\delta) t}
$$

Setting $I_{2}=c_{3}$, we find

$$
\begin{gathered}
\frac{c^{-\sigma} e^{-\rho t}}{1-\sigma}\left[(\rho+\pi-\pi \sigma) k-\sigma c-\beta \gamma(1-\sigma)\left(\frac{u h}{k}\right)^{1-\beta} k\right. \\
\left.+\frac{(1-\beta) \gamma}{\delta}(\rho-\delta+\delta \sigma)\left(\frac{k}{u h}\right)^{\beta} h\right]=c_{3}
\end{gathered}
$$

and this gives an expression for human capital

$$
h(t)=\frac{\left(\frac{c_{3}(1-\sigma)}{\lambda \mathrm{e}^{-\rho t}}-(\rho+\pi-\pi \sigma) k+\sigma c+\beta \gamma(1-\sigma) z^{1-\beta} k\right) \delta z^{\beta}}{\gamma(1-\beta)(\rho-\delta+\delta \sigma)} .
$$

The transversality condition for $h$ requires to choose $c_{3}=0$ and $\delta<\rho+$ $\delta \sigma$. Finally, the variable $u$, the fraction of labor devoted to the production of physical capital can be determined from $u=z k / h$ and it simplifies to

$$
u(t)=\frac{\delta^{-1} \gamma(1-\beta)(\rho-\delta+\delta \sigma)\left(k_{0} /\left(c_{0} z_{0}^{\frac{\beta-\sigma}{\sigma}}\right)-F(t)\right)}{\left[\beta \gamma(1-\sigma)-(\rho+\pi-\pi \sigma) z^{\beta-1}\right]\left(\frac{k_{0}}{c_{0} z_{0}^{\frac{\beta-\sigma}{\sigma}}}-F(t)\right)+\sigma z^{\beta-\frac{\beta}{\sigma} e^{-\left(\frac{\delta+\pi-\pi \beta}{\beta}-\frac{\delta-\rho}{\sigma}\right) t}} .}
$$


This completes the solution. We then apply the initial conditions $h(0)=h_{0}$, $u_{0}=\frac{z_{0} k_{0}}{h_{0}}$ and we summarize the solutions for all variables as follows:

$$
\begin{aligned}
& c(t)=c_{0} z_{0}^{\frac{\beta}{\sigma}} e^{-\frac{(\rho-\delta)}{\sigma} t} z^{-\frac{\beta}{\sigma}}, \quad k(t)=\left(\frac{k_{0}}{c_{0} z_{0}^{\frac{\beta-\sigma}{\sigma}}}-F(t)\right) c_{0} z_{0}^{\frac{\beta}{\sigma}} z(t)^{-1} e^{\frac{(\delta+\pi-\pi \beta)}{\beta} t}, \\
& h(t)=\frac{h_{0}}{z_{0}\left[\sigma c_{0} z_{0}^{\beta-1}-(\rho+\pi-\pi \sigma) k_{0} z_{0}^{\beta-1}+\beta \gamma(1-\sigma) k_{0}\right]}\left[\sigma c_{0} z_{0}^{\frac{\beta}{\sigma}} e^{-\frac{(\rho-\delta)}{\sigma} t} z^{-\frac{\beta}{\sigma}+\beta}\right. \\
& \left.+\left(\beta \gamma(1-\sigma)-(\rho+\pi-\pi \sigma) z^{\beta-1}\right)\left(\frac{k_{0}}{c_{0} z_{0}^{\frac{\beta-\sigma}{\sigma}}}-F(t)\right) c_{0} z_{0}^{\frac{\beta}{\sigma}} e^{\frac{(\delta+\pi-\pi \beta)}{\beta} t}\right], \\
& u(t)=\frac{u_{0}}{k_{0}}\left[\sigma c_{0} z_{0}^{\beta-1}-(\rho+\pi-\pi \sigma) k_{0} z_{0}^{\beta-1}+\beta \gamma(1-\sigma) k_{0}\right] \\
& \times \frac{\left(k_{0} /\left(c_{0} z_{0}^{\frac{\beta-\sigma}{\sigma}}\right)-F(t)\right)}{\left[\beta \gamma(1-\sigma)-(\rho+\pi-\pi \sigma) z^{\beta-1}\right]\left(\frac{k_{0}}{c_{0} z_{0}^{\frac{\beta-\sigma}{\sigma}}}-F(t)\right)+\sigma z^{\beta-\frac{\beta}{\sigma}} e^{-\left(\frac{\delta+\pi-\pi \beta}{\beta}-\frac{\delta-\rho}{\sigma}\right) t}}, \\
& \lambda(t)=c_{0}^{-\sigma} z_{0}^{-\beta} e^{(\rho-\delta) t} z^{\beta}, \quad \mu(t)=c_{1} e^{(\rho-\delta) t},
\end{aligned}
$$

where

$$
\begin{aligned}
& F(t)=\int_{0}^{t} z(t)^{\frac{\sigma-\beta}{\sigma}} e^{-\left(\frac{\delta+\pi-\pi \beta}{\beta}-\frac{\delta-\rho}{\sigma}\right) t} d t \\
& z(t)=\frac{z^{*} z_{0}}{\left[\left(z^{* 1-\beta}-z_{0}^{1-\beta}\right) e^{-\frac{(1-\beta)(\delta+\pi)}{\beta} t}+z_{0}^{1-\beta}\right]^{\frac{1}{1-\beta}}}, \\
& \lim _{t \rightarrow \infty} F(t)=\frac{k_{0}}{c_{0} z_{0}^{\frac{\beta-\sigma}{\sigma}}}, \quad \rho<\delta<\rho+\delta \sigma, \quad \frac{\delta+\pi-\pi \beta}{\beta}-\frac{\delta-\rho}{\sigma}>0 \\
& c_{0} z_{0}^{\frac{\beta}{\sigma}}=\left(\frac{c_{1} \delta}{(1-\beta) \gamma}\right)^{-\frac{1}{\sigma}}, \quad z^{*}=\left(\frac{\beta \gamma}{\delta+\pi}\right)^{\frac{1}{\beta-1}}, \\
& \frac{\gamma(1-\beta)(\rho-\delta+\delta \sigma)}{\delta}=\frac{u_{0}}{k_{0}}\left[\sigma c_{0} z_{0}^{\beta-1}-(\rho+\pi-\pi \sigma) k_{0} z_{0}^{\beta-1}+\beta \gamma(1-\sigma) k_{0}\right] .
\end{aligned}
$$

The first integrals $I_{1}$ and $I_{2}$ yield two solutions of the dynamical system of ODEs (2.10)-(2.15) given in equations (3.11) and (3.21) for fairly general values of $\sigma$ and $\beta$. It is straight forward to show that

$$
\lim _{t \rightarrow \infty} u(t)=u^{*}
$$

\section{Closed-form solution for the Lucas-Uzawa model under fairly general values of parameters via $I_{1}$}

Now we show how one can utilize only one first integral $I_{1}$ to derive the same closed-from solution as in the existing literature which was also derived by Chilarescu [3] via the classical approach. By setting $I_{1}=c_{1}$, we will arrive at equations (3.1)-(3.4).

Here also the following two scenarios will arise: Case I: $c_{2}=0$, Case II: $c_{2} \neq 0$. 
Equations (3.5)-(3.10) for variables $c(t), k(t), \lambda(t)$ and $\mu(t)$ will follow from the previous section. Now instead of utilizing the second first integral, we proceed as follows to derive closed form solutions for $h(t)$ and $u(t)$.

Equation (2.15) simplifies to

$$
\frac{\dot{u}}{u}=\frac{\delta-\rho-\delta \sigma}{\sigma}+\delta u
$$

Equation (4.1) gives

$$
u(t)=\frac{(\delta-\rho-\delta \sigma) / \sigma}{a_{2}\left(\frac{\delta-\rho-\delta \sigma}{\sigma}\right) e^{-\frac{\delta-\rho-\delta \sigma}{\sigma} t}-\delta} .
$$

Finally, $h=z k / u$ gives

$$
h(t)=\frac{a_{2}\left(\frac{\delta-\rho-\delta \sigma}{\sigma}\right) e^{-\frac{\delta-\rho-\delta \sigma}{\sigma} t}-\delta}{(\delta-\rho-\delta \sigma) / \sigma} z^{*} k_{0} e^{-\frac{(\rho-\delta)}{\sigma} t} .
$$

The transversality condition (2.7) for $h$ is satisfied provided $\delta<\rho+\delta \sigma$ and $a_{2}=0$. Thus we arrive at the same solution for all variables as given by equation (3.11).

For $a_{2} \neq 0$, we will follow same procedure as described in previous section to derive equations (3.12)-(3.20). Substituting $c(t)$ and $k(t)$ from equations (3.14) and (3.18) into equation (2.15), we have

$$
\frac{\dot{u}}{u}=\frac{(\delta+\pi)(1-\beta)}{\beta}-\frac{z(t)^{\frac{\sigma-\beta}{\sigma}} e^{-\left(\frac{\delta+\pi-\pi \beta}{\beta}-\frac{\delta-\rho}{\sigma}\right) t}}{k_{0} /\left(c_{0} z_{0}^{\frac{\beta-\sigma}{\sigma}}\right)-F(t)}+\delta u .
$$

The solution of equation (4.2) with initial condition $u(0)=u_{0}$ is given by (see Chilarescu [3])

$$
u(t)=\frac{\frac{(\delta+\pi)(1-\beta)}{\beta} u_{0}\left[k_{0} /\left(c_{0} z_{0}^{\frac{\beta-\sigma}{\sigma}}\right)-F(t)\right]}{H(t)},
$$

where

$$
\begin{aligned}
H(t)= & {\left[\left(\frac{(\delta+\pi)(1-\beta)}{\beta}+\delta u_{0}\right) \frac{k_{0}}{c_{0} z_{0}^{(\beta-\sigma) / \sigma}}-\delta u_{0} G(t)\right] e^{-\frac{(\delta+\pi)(1-\beta)}{\beta} t} } \\
& -\delta u_{0}\left[\frac{k_{0}}{c_{0} z_{0}^{(\beta-\sigma) / \sigma}}-F(t)\right] \\
G(t)= & \int_{0}^{t} z(t)^{\frac{\sigma-\beta}{\sigma}} e^{-\frac{\delta \sigma-\delta-\rho}{\sigma} t} d t .
\end{aligned}
$$

The solution (4.3) holds provided

$$
\lim _{t \rightarrow \infty}\left[\left(\frac{(\delta+\pi)(1-\beta)}{\beta}+\delta u_{0}\right) \frac{k_{0}}{c_{0} z_{0}^{(\beta-\sigma) / \sigma}}-\delta u_{0} G(t)\right]=0,
$$


and this can be re-written as

$$
\lim _{t \rightarrow \infty} G(t)=\frac{(\delta+\pi)(1-\beta) / \beta+\delta u_{0}}{\delta u_{0}} \lim _{t \rightarrow \infty} F(t),
$$

where $\lim _{t \rightarrow \infty} F(t)$ is given in (3.19). The variable $h$ can be determined from $h=z k / u$ and is given by

$$
\begin{aligned}
& h(t)=\left[\left(\left(\frac{(\delta+\pi)(1-\beta)}{\beta}+\delta u_{0}\right) \frac{k_{0}}{c_{0} z_{0}^{(\beta-\sigma) / \sigma}}-\delta u_{0} G(t)\right) e^{-\frac{(\delta+\pi)(1-\beta)}{\beta} t}\right. \\
& \left.-\delta u_{0}\left(\frac{k_{0}}{c_{0} z_{0}^{(\beta-\sigma) / \sigma}}-F(t)\right)\right] \frac{c_{0} z_{0}^{\beta / \sigma}}{\frac{(\delta+\pi)(1-\beta)}{\beta} u_{0}} e^{\frac{(\pi+\delta-\pi \beta)}{\beta} t} .
\end{aligned}
$$

We can summarize the solutions for all variables as follows:

$$
\begin{aligned}
c(t) & =c_{0} z_{0}^{\frac{\beta}{\sigma}} e^{-\frac{(\rho-\delta)}{\sigma} t} z^{-\frac{\beta}{\sigma}}, \quad k(t)=\left(\frac{k_{0}}{c_{0} z_{0}^{(\beta-\sigma) / \sigma}}-F(t)\right) c_{0} z_{0}^{\frac{\beta}{\sigma}} z(t)^{-1} e^{\frac{(\pi+\delta-\pi \beta)}{\beta} t}, \\
h(t) & =\left[\left(\frac{(\delta+\pi)(1-\beta)}{\beta} \frac{k_{0}}{c_{0} z_{0}^{(\beta-\sigma) / \sigma}}+\frac{\delta u_{0} k_{0}}{c_{0} z_{0}^{(\beta-\sigma) / \sigma}}-\delta u_{0} G(t)\right) e^{-\frac{(\delta+\pi)(1-\beta)}{\beta} t}\right. \\
& \left.-\delta u_{0}\left(\frac{k_{0}}{c_{0} z_{0}^{(\beta-\sigma) / \sigma}}-F(t)\right)\right] \frac{c_{0} z_{0}^{\beta / \sigma}}{\frac{(\delta+\pi)(1-\beta)}{\beta} u_{0}} e^{\frac{(\pi+\delta-\pi \beta)}{\beta} t}, \\
u(t) & =\frac{\frac{(\delta+\pi)(1-\beta)}{\beta} u_{0}\left[k_{0} /\left(c_{0} z_{0}^{\frac{\beta-\sigma}{\sigma}}\right)-F(t)\right]}{H(t)} \\
\lambda(t) & =c_{0}^{-\sigma} z_{0}^{-\beta} e^{(\rho-\delta) t} z^{\beta}, \quad \mu(t)=c_{1} e^{(\rho-\delta) t},
\end{aligned}
$$

where

$$
\begin{aligned}
& \rho<\delta<\rho+\delta \sigma, \quad \frac{\delta+\pi-\pi \beta}{\beta}-\frac{\delta-\rho}{\sigma}>0 \\
& F(t)=\int_{0}^{t} z(t)^{\frac{\sigma-\beta}{\sigma}} e^{-\left(\frac{\delta+\pi-\pi \beta}{\beta}-\frac{\delta-\rho}{\sigma}\right) t} d t, \quad G(t)=\int_{0}^{t} z(t)^{\frac{\sigma-\beta}{\sigma}} e^{-\frac{\delta \sigma-\delta+\rho}{\sigma} t} d t \\
& z(t)=\frac{z^{*} z_{0}}{\left[\left(z^{* 1-\beta}-z_{0}^{1-\beta}\right) e^{-\frac{(1-\beta)(\delta+\pi)}{\beta} t}+z_{0}^{1-\beta}\right]^{\frac{1}{1-\beta}}}, \\
& c_{0} z_{0}^{\frac{\beta}{\sigma}}=\left(\frac{c_{1} \delta}{(1-\beta) \gamma}\right)^{-\frac{1}{\sigma}}, \quad \lim _{t \rightarrow \infty} F(t)=\frac{k_{0}}{c_{0} z_{0}^{(\beta-\sigma) / \sigma}}, \\
& \lim _{t \rightarrow \infty}\left[\left(\frac{(\delta+\pi)(1-\beta)}{\beta}+\delta u_{0}\right) \frac{k_{0}}{\left.c_{0} z_{0}^{(\beta-\sigma) / \sigma}-\delta u_{0} G(t)\right]=0}\right. \\
& \lim _{t \rightarrow \infty} G(t)=\frac{\left(\frac{(\delta+\pi)(1-\beta)}{\beta}+\delta u_{0}\right)}{\delta u_{0}} \lim _{t \rightarrow \infty} F(t), \quad z^{*}=\left(\frac{\beta \gamma}{\delta+\pi}\right)^{\frac{1}{\beta-1}} .
\end{aligned}
$$

The closed form solution (4.6) derived by only utilizing $I_{1}$ is exactly the same as derived by Chilarescu [3] via the classical approach. 


\section{Comparison of Closed-form Solutions for the Lucas- Uzawa model}

The closed form solutions of the Lucas-Uzawa model of economic growth have been derived in the literature by using both the newly developed partial Hamiltonian approach and the classical approach. The partial Hamiltonian approach utilizes Lie group theoretical techniques to construct a closed-form solution. Using the partial Hamiltonian methodology, we have established three sets of closed form solutions (3.11), (3.21) and (4.6) for the Lucas-Uzawa model with no parameter restrictions. For the case $z=z^{*}$ case only one solution arises which is given in (3.11) whereas for the case $z \neq z^{*}$ case we obtained two solutions which are given in (3.21) and (4.6). We have shown how first integrals derived via the partial Hamiltonian approach can be utilized to construct multiple closed form solutions for the Lucas-Uzawa model. Thus there exist three sets of closed form solutions (3.11), (3.21) and (4.6) for the Lucas-Uzawa model with no parameter restrictions.

\subsection{Comparison with Chilarescu [3]}

Chilarescu [3] made a significant contribution by utilizing the classical approach to obtain two closed form solutions of the form (3.11) and (4.6). We have shown in previous sections that the solution (3.11) can also be constructed by utilizing either one first integral $I_{1}$ or by using two first integrals $I_{1}$ and $I_{2}$. For the case where $z \neq z^{*}$, we have obtained the second solution (4.6) found by Chilarescu [3] and this solution was obtained by utilizing only one first integral $I_{1}$. Using our partial Hamiltonian methodology, we also arrive at an additional new solution (3.21) in the case where $z \neq z^{*}$ by utilizing the two first integrals $I_{1}$ and $I_{2}$.

A comparison of the closed-form solutions (3.21) and (4.6) shows that the expressions for consumption $c$, physical capital stock $k$, and the costate variables $\lambda$ and $\mu$ are the same in both solutions. On the other hand, the expressions for the fraction of labor devoted to physical capital, $u$, and the level of human capital, $h$, are different in our newly obtained solution. This is important in the context of economic growth theory because it shows that an economy can either be in an equilibrium with a higher level of human capital or in an equilibrium with a lower level of human capital. Another important result is that the previously obtained closed form solution (4.6) involves two numerically computable functions $F(t)$ and $G(t)$ whereas our newly derived closed form solution (3.21) involves only one numerically computable function $F(t)$. So our newly derived closed form solution (3.21) which was obtained from the partial Hamiltonian approach is fundamentally different and is also in a simpler form than the previously obtained solution (4.6).

\subsection{Comparison with Boucekkine and Ruiz-Tamarit [1]}

Boucekkine and Ruiz-Tamarit [1] were the first to use the classical approach to derive elegant closed form solutions and they obtained one solution under no parameter restrictions which is similar to (4.6). Chilarescu [3] then utilized 
the classical approach to provide two solutions with no restrictions on the parameters which are the same as the solutions in (3.11) and (4.6). So in both [3] and [1], the Lucas-Uzawa model was solved by the classical approach. For the closed-form solution (4.6), the only difference in these two works is the evaluation of the functions $F(t)$ and $G(t)$. In [3], the functions $F(t)$ and $G(t)$ were computed by numerical simulations whereas in [1] these type of functions were solved in terms of Gaussian Hypergeometric functions. With the aid of numerical simulations, it is well-demonstrated by Chilarescu [3], that the transitional dynamics of variables for solution (4.6) were same as derived by Boucekkine and Ruiz-Tamarit [1].

It is important to note that the partial Hamiltonian approach also provides one additional solution given in (3.21) which is new to the literature. Moreover, under specific restriction $\sigma=\frac{\beta(\rho+\pi)}{2 \pi \beta-\delta+\delta \beta-\pi}$ on parameters another first integral was established and another set of closed form solution was derived in Naz et al [9]. It was also shown that the closed-form solution under this specific parameter restriction converges to the balanced growth path. The closed form solution under this specific restriction was not derived before in literature. Thus partial Hamiltonian approach provides four sets of closed-from solutions: three under no parameter restriction and fourth is under parameter restriction $\sigma=\frac{\beta(\rho+\pi)}{2 \pi \beta-\delta+\delta \beta-\pi}$ whereas classical approach provides olny two closed-form solutions.

\subsection{Convergence to balanced growth path}

The discussion of convergence of closed-form (3.11) and (4.6) is well presented in detail in Chilarescu [3]. Boucekkine and Ruiz-Tamarit [1] analyzed closedform solution (4.6). Naz et al [9] provided detailed analysis and convergence to balanced growth path for the closed-from solution under specific restriction $\sigma=\frac{\beta(\rho+\pi)}{2 \pi \beta-\delta+\delta \beta-\pi}$ on parameters. We provide here a detailed analysis of newly derived closed-form solution (3.21). We prove that our newly derived solution (3.21) satisfies all properties stated in Proposition 1 of Chilarescu [3]. It is straight forward to show, for the closed form solutions (3.21) that

$$
\lim _{t \rightarrow \infty} u(t)=u^{*}=\frac{\rho-\delta+\delta \sigma}{\delta \sigma} .
$$

It is worthy to mention here that l'Hôpital rule is applied to establish $\lim t \rightarrow \infty$ of the fraction of labor devoted to the production of physical capital $u(t)$. We find the ratio of consumption to the capital stock for economy

$$
\frac{c(t)}{k(t)}=\frac{z(t)^{\frac{\sigma-\beta}{\sigma}} e^{-\left(\frac{\delta+\pi-\pi \beta}{\beta}-\frac{\delta-\rho}{\sigma}\right) t}}{\frac{k_{0}}{c_{0} z_{0}^{(\beta-\sigma) / \sigma}}-F(t)}, \quad \lim _{t \rightarrow \infty} \frac{c(t)}{k(t)}=\frac{\delta+\pi-\pi \beta}{\beta}-\frac{\delta-\rho}{\sigma} .
$$

Next, we find the ratio of the capital stock to the fraction of labor for economy

$$
\lim _{t \rightarrow \infty} \frac{k(t)}{h(t)}=\lim _{t \rightarrow \infty} \frac{u(t)}{z(t)}=\frac{\rho-\delta+\delta \sigma}{\delta \sigma}\left(\frac{\beta \gamma}{\delta+\pi}\right)^{\frac{1}{\beta-1}}
$$


The growth rates of all the variables for the closed-form solution (3.21) after simplifications are

$$
\begin{aligned}
\frac{\dot{c}}{c}= & \frac{\delta-\rho}{\sigma}-\frac{\beta}{\sigma} \frac{\dot{z}}{z}, \\
\frac{\dot{k}}{k}= & \frac{\pi+\delta-\pi \beta}{\beta}-\frac{z(t)^{\frac{\sigma-\beta}{\sigma}} e^{-\left(\frac{\delta+\pi-\pi \beta}{\beta}-\frac{\delta-\rho}{\sigma}\right) t}}{k_{0} /\left(c_{0} z_{0}^{\frac{\sigma-\beta}{\beta}}\right)-F(t)}-\frac{\dot{z}}{z}, \\
\frac{\dot{h}}{h}= & \frac{\sigma z(t)^{\beta-1}}{\sigma z(t)^{\beta-1}+\left[\beta \gamma(1-\sigma)-(\rho+\pi-\pi \sigma) z(t)^{\beta-1}\right] \frac{k(t)}{c(t)}} \frac{\dot{c}}{c} \\
& +\frac{\sigma \beta z(t)^{\beta-1} \frac{c(t)}{k(t)}-(\rho+\pi-\pi \sigma)(\beta-1) z(t)^{\beta-1}}{\sigma z(t)^{\beta-1} \frac{c(t)}{k(t)}+\beta \gamma(1-\sigma)-(\rho+\pi-\pi \sigma) z(t)^{\beta-1}} \frac{\dot{z}}{z} \\
& +\frac{\left[\beta \gamma(1-\sigma)-(\rho+\pi-\pi \sigma) z(t)^{\beta-1}\right]}{\sigma z(t)^{\beta-1} \frac{c(t)}{k(t)}+\beta \gamma(1-\sigma)-(\rho+\pi-\pi \sigma) z(t)^{\beta-1}} \frac{\dot{z}}{z} \\
& +\frac{\beta \gamma(1-\sigma)-(\rho+\pi-\pi \sigma) z(t)^{\beta-1}}{\sigma z(t)^{\beta-1} \frac{c(t)}{k(t)}+\beta \gamma(1-\sigma)-(\rho+\pi-\pi \sigma) z(t)^{\beta-1}} \frac{\dot{k}}{k}, \\
\frac{\dot{u}}{u}= & \frac{\dot{k}}{k}-\frac{\dot{h}}{h}+\frac{\dot{z}}{z}, \quad \frac{\dot{\lambda}}{\lambda}=\rho-\delta+\beta \frac{\dot{z}}{z}, \quad \frac{\dot{\mu}}{\mu}=\rho-\delta,
\end{aligned}
$$

where

$$
\frac{\dot{z}}{z}=\frac{(\pi+\delta)\left(z^{* 1-\beta}-z_{0}^{1-\beta}\right) e^{-\frac{(1-\beta)(\pi+\delta)}{\beta} t}}{\beta\left(z_{0}^{1-\beta}+\left(z^{* 1-\beta}-z_{0}^{1-\beta}\right) e^{-\frac{(1-\beta)(\pi+\delta)}{\beta} t}\right)} .
$$

Now we analyze the dynamic growth rates provided in (5.1). First we observe that as $t \rightarrow \infty, \frac{\dot{z}}{z}$ approaches to zero which shows that the rate of growth of $z(t)$ decreases asymptotically as we approach the steady state. The growth rates of consumption, $c$, physical capital, $k$, and human capital, $h$, decrease over time and approach $\frac{\delta-\rho}{\sigma}$ as $t \mapsto \infty$. Also, the growth rate of the fraction of labor allocated to the production of physical capital, $u$, approaches zero as $t \mapsto \infty$ whereas the growth rates of both costate variables, $\lambda$ and $\mu$, equal $(\rho-\delta)$ as $t \mapsto \infty$. In the long run our newly derived solution (3.21) solutions converge to the same balanced growth path as solutions (3.11) and (4.6). This is important in the context of economic growth theory and gives rise to multiple equilibria. This establishes the convergence of our newly derived closed-form solution (3.21).

\section{Closed-from solution for $\sigma=\beta$ case}

In this Section, we consider the special case when $\sigma=\beta$ which is commonly discussed in economic growth theory. We obtained two closed-form solutions for Lucas-Uzawa model under restriction $\sigma=\beta$ whether we utilize only $I_{1}$ or we utilize both $I_{1}$ and $I_{2}$. 
For $z=z^{*}$ case, the closed-form solution for all variables is obtained by taking $\sigma=\beta$ in (3.11) and is given by

$$
\begin{aligned}
& c(t)=c_{0} e^{-\frac{(\rho-\delta)}{\beta} t}, \quad k(t)=k_{0} e^{-\frac{(\rho-\delta)}{\beta} t}, \quad u(t)=\frac{\rho-\delta+\delta \beta}{\delta \beta}=u^{*}, \\
& h(t)=h_{0} e^{-\frac{(\rho-\delta)}{\beta} t}, \quad \lambda(t)=c_{0}^{-\beta} e^{(\rho-\delta) t}, \quad \mu=c_{1} e^{(\rho-\delta) t}, \quad z^{*}=\left(\frac{\delta+\pi}{\beta \gamma}\right)^{\frac{1}{1-\beta}},
\end{aligned}
$$

provided $\delta<\rho+\delta \beta, c_{0}=\left(\frac{(1-\beta) \gamma}{c_{1} \delta z^{* \beta}}\right)^{\frac{1}{\beta}}, \frac{c_{0}}{k_{0}}=\frac{\rho+\pi(1-\beta)}{\beta}>0, h_{0}=\frac{z^{*} k_{0}}{u^{*}}$.

Next, we take $\sigma=\beta$ in (3.21), the variable $c(t)$ takes following form:

$$
c(t)=c_{0} z_{0} e^{-\frac{(\rho-\delta)}{\beta} t} z(t)^{-1} .
$$

The equation (3.17) for $F(t)$ simplifies to

$$
F(t)=\frac{\beta}{\pi-\pi \beta+\rho}\left(1-e^{-\frac{(\pi-\pi \beta+\rho)}{\beta} t}\right)
$$

and (3.19) yields

$$
\frac{c_{0}}{k_{0}}=\frac{\rho+\pi(1-\beta)}{\beta} .
$$

The variable $k(t)$ takes following form:

$$
k(t)=k_{0} z_{0} e^{-\frac{(\rho-\delta)}{\beta} t} z(t)^{-1}
$$

and satisfies the transversality condition provided $\delta<\rho+\delta \beta$. After some simplifications, the variables $h(t)$ and $u(t)$ in (3.21) take following form:

$$
h(t)=h_{0} e^{-\frac{(\rho-\delta)}{\beta} t}
$$

and $u(t)=u_{0}$. The condition

$$
\frac{\gamma(1-\beta)(\rho-\delta+\delta \sigma)}{\delta}=\frac{u_{0}}{k_{0}}\left[\sigma c_{0} z_{0}^{\beta-1}-(\rho+\pi-\pi \sigma) k_{0} z_{0}^{\beta-1}+\beta \gamma(1-\sigma) k_{0}\right]
$$

provides $u_{0}=\frac{\rho-\delta+\delta \beta}{\delta \beta}$.

For $z \neq z^{*}$ case, the closed-form solution for all variables is summarized as follows:

$$
\begin{aligned}
& c(t)=c_{0} z_{0} e^{-\frac{(\rho-\delta)}{\beta} t} z(t)^{-1}, k(t)=k_{0} z_{0} e^{-\frac{(\rho-\delta)}{\beta} t} z(t)^{-1}, h(t)=h_{0} e^{-\frac{(\rho-\delta)}{\beta} t}, \\
& u(t)=\frac{\rho-\delta+\delta \beta}{\delta \beta}=u^{*}, \quad \lambda(t)=c_{0}^{-\beta} z_{0}^{-\beta} e^{(\rho-\delta) t} z(t)^{\beta}, \quad \mu(t)=c_{1} e^{(\rho-\delta) t},
\end{aligned}
$$

provided

$$
\delta<\rho+\delta \beta, \quad c_{0}=\left(\frac{(1-\beta) \gamma}{c_{1} \delta z_{0}^{\beta}}\right)^{\frac{1}{\beta}}, \quad \frac{c_{0}}{k_{0}}=\frac{\rho+\pi(1-\beta)}{\beta}>0,
$$




$$
h_{0}=\frac{z_{0} k_{0}}{u^{*}}, \quad z(t)=\frac{z^{*} z_{0}}{\left[\left(z^{* 1-\beta}-z_{0}^{1-\beta}\right) e^{-\frac{(1-\beta)(\delta+\pi)}{\beta} t}+z_{0}^{1-\beta}\right]^{\frac{1}{1-\beta}}} .
$$

Next, we take $\sigma=\beta$ in (4.6). In (4.6), only the expressions for $u(t)$ and $h(t)$ are different from (3.21). Equations (4.4) and (4.5) for $\sigma=\beta$ case reduces to the following form:

$$
\begin{aligned}
& G(t)=\frac{\beta}{\delta \beta-\delta+\rho}\left(1-e^{-\frac{(\delta \beta-\delta+\rho)}{\beta} t}\right) \\
& \left(\frac{(\delta+\pi)(1-\beta)}{\beta}+\delta u_{0}\right) \frac{k_{0}}{c_{0}}=\frac{\delta u_{0} \beta}{\delta \beta-\delta+\rho},
\end{aligned}
$$

where $\frac{c_{0}}{k_{0}}$ is given in (6.2). With the aid of equations, (6.4) and (6.5), the equations for the variables $u(t)$ and $h(t)$ reduce to same equations as presented in $(6.3)$.

We conclude that for the special case $\sigma=\beta$, both solutions (3.21) and (4.6) reduces to same solution (6.3). This also verifies the correctness of our newly derived solution (3.21). It is important to mention here that $\sigma=\beta$ assumption leads to the constancy of $\frac{c(t)}{k(t)}$ and we obtain less solutions in this case. In the previous literature only the solution (6.3) had been reported. It is important to mention here that the (6.1) directly follows from (6.3) by setting $z=z^{*}$ so it is sufficient to just mention (6.3) (see e.g $[6,7]$ ).

\subsection{Comparison with Marsiglio and La Torre [7]}

We can compare the closed-form solution (6.3) with the previous literature [7]. In [7], the population dynamics were discussed for three different types of population growth: exponential, logistic and von Bertalanffy. If we simplify the model by Marsiglio and La Torre [7] by taking $N=1$ and $n=0$ to normalize the effect of population growth to zero and if we set $\pi=0$ in our model then we find that both models take the same form and the only difference is in terms of the notations used for different parameters. The solution presented in equations (33)-(36) of Marsiglio and La Torre [7] is exactly the same as the closed-form solution provided in (6.3). This can be established by using the expression for $z(t)$ which provides the alternative form of the solution (6.3) as

$$
\begin{aligned}
& k(t)=e^{-\left(\frac{\rho+\pi}{\beta}\right) t}\left[k_{0}^{1-\beta}+\frac{\gamma u^{1-\beta} h_{0}^{1-\alpha}}{(\rho+\pi) / \beta+\delta-\delta u}\left(e^{(1-\beta)((\rho+\pi) / \beta+\delta-\delta u) t}-1\right)\right]^{\frac{1}{1-\beta}}, \\
& c(t)=\frac{c_{0}}{k_{0}} k(t), \quad h(t)=h_{0} e^{\delta(1-u) t}, \quad u(t)=\frac{\rho-\delta+\delta \beta}{\delta \beta}=u^{*}, \\
& \lambda(t)=c_{0}^{-\beta} z_{0}^{-\beta} e^{(\rho-\delta) t} z(t)^{\beta}, \quad \mu(t)=c_{1} e^{(\rho-\delta) t}, \quad \frac{c_{0}}{k_{0}}=\frac{\rho+\pi(1-\beta)}{\beta},
\end{aligned}
$$

which is exactly the same as presented in equations (33)-(36) of Marsiglio and La Torre [7] if the population growth is normalized to zero ${ }^{1}$.

\footnotetext{
${ }^{1} \operatorname{In}[7], \delta=B, \beta=\alpha, \gamma=A, \pi=0$
} 


\subsection{Comparison with Marsiglio and La Torre [6]}

We can also compare the closed-form solution (6.3) with another solution found in the previous literature [6] in which a closed-form solution was established by using the dynamic programming technique. By setting $N(t)=1, \delta_{H}=0$ for $\beta=1-\alpha$, the model by Marsiglio and La Torre [6] reduces to same model as presented here just there is difference in terms of notations used for different parameters. The solution presented by equations (11)-(14) reduces to the form (6.6) provided $N(t)=1, \delta_{H}=0$ for $\beta=1-\alpha$.

\section{Conclusions}

In this paper we establish closed-form solutions for the Lucas-Uzawa model with the aid of the partial Hamiltonian approach and then compare our results with those derived by the classical approach. The partial Hamiltonian approach provides two first integrals [9] in the case where there are no parameter restrictions. We utilize these two first integrals to construct closed form solutions for all the economic variables in the model for two different scenarios: (i) $z=z^{*}$ and (ii) $z \neq z^{*}$ where $z(t)=\frac{h(t) u(t)}{k(t)}$.

For the $z=z^{*}$ case, both the partial Hamiltonian approach and the classical approach provide one solution. For the $z \neq z^{*}$ case, the classical approach yields one solution while the the partial Hamiltonian approach provides the same solution as well as one additional solution which is completely new to the literature. In the newly obtained solution, the expressions for the levels of consumption and capital stock are equal to those found in the older solution, while the amount of labor allocated to the production of physical capital and the level of human capital are different from those values found in the older solution. The existence of three closed form solutions is new to the literature on economic growth. We also show that the economic equilibria all converge to the same long run balanced growth path. We have also analyzed the special case $\sigma=\beta$ and obtained two sets of closed-form solutions. In addition to this, we also present a detailed comparison of our derived results with the previous literature for the $\sigma \neq \beta$ case as well as for the $\sigma=\beta$ case.

\section{References}

[1] R. Boucekkine and J.R. Ruiz Tamarit. Special functions for the study of economic dynamics: The case of the Lucas-Uzawa model. Journal of Mathematical Economics, 44(1):33-54, 2008. https://doi.org/10.1016/j.jmateco.2007.05.001.

[2] A.C. Chiang. Elements of dynamic optimization. McGraw-Hill, Newyork, 1992.

[3] C. Chilarescu. On the existence and uniqueness of solution to the lucas-uzawa model. Economic Modelling, 28(1-2):109-117, 2011. https://doi.org/10.1016/j.econmod.2010.09.019.

[4] V. Dorodnitsyn and R. Kozlov. Invariance and first integrals of continuous and discrete hamiltonian equations. Journal of Engineering Mathematics, 66(1):253270, 2010. https://doi.org/10.1007/s10665-009-9312-0. 
[5] R.E. Lucas. On the mechanics of economic development. Journal of Monetary Economics, 22(1):3-42, 1988. https://doi.org/10.1016/0304-3932(88)90168-7.

[6] S. Marsiglio and D. La Torre. A note on demographic shocks in a multi-sector growth model. Economics Bulletin, 32(3):2293-2299, 2012.

[7] S. Marsiglio and D. La Torre. Population dynamics and utilitarian criteria in the Lucas-Uzawa model. Economic Modelling, 29(4):1197-1204, 2012. https://doi.org/10.1016/j.econmod.2012.01.016.

[8] R. Naz. The applications of the partial Hamiltonian approach to mechanics and other areas. International Journal of Non-linear mechanics, 86:1-6, 2016. https://doi.org/10.1016/j.ijnonlinmec.2016.07.009.

[9] R. Naz, A. Chaudhry and F.M. Mahomed. Closed-form solutions for the LucasUzawa model of economic growth via the partial Hamiltonian approach. Communications in Nonlinear Science and Numerical Simulation, 30(1-3):299-306, 2016. https://doi.org/10.1016/j.cnsns.2015.06.033.

[10] R. Naz, F.M. Mahomed and A. Chaudhry. A partial Hamiltonian approach for current value Hamiltonian systems. Communications in Nonlinear Science and Numerical Simulation, 19(10):3600-3610, 2014. https://doi.org/10.1016/j.cnsns.2014.03.023.

[11] H. Uzawa. Optimum technical change in an aggregative model of economic growth. International Economic Review, 6(1):18-31, 1965. https://doi.org/10.2307/2525621. 